\title{
Genetic structure of Octopus minor around Chinese waters as indicated by nuclear DNA variations (Mollusca, Cephalopoda)
}

\author{
Faiz Muhammad',2, Zhen-ming Lü', Liqin Liu', Li Gong', Xun Du', \\ Muhammad Shafi ${ }^{3}$, Hubdar Ali Kaleri ${ }^{4}$
}

I National Engineering Research Center of Marine Facilities Aquaculture, College of Marine Sciences and Technology, Zhejiang Ocean University 2 Center of Excellence in Marine Biology, University of Karachi 3 Lasbella University of Agriculture, Water and Marine Sciences 4 Department of Animal Science and Aquaculture, Dalhousie University, Canada

Corresponding author: Zhen-ming Lü (nblzmnb@163.com)

Academic editor: P. Stoev | Received 6 February 2018 | Accepted 29 May 2018 | Published 17 July 2018

http://zoobank.org/CB017F1A-193C-481A-8C93-513A8D3C614F

Citation: Muhammad F, Lü Z-m, Liu L, Gong L, Du X, Shafi M, Kaleri HA (2018) Genetic structure of Octopus minor around Chinese waters as indicated by nuclear DNA variations (Mollusca, Cephalopoda). ZooKeys 775: 1-14. https://doi.org/10.3897/zookeys.775.24258

\begin{abstract}
Octopus minor is an economically important resource commonly found in Chinese coastal waters. The nuclear gene (RD and $\mathrm{ODH}$ ) approach of investigation has not reported in this species. Rhodopsin $(\mathrm{RD})$ and octopine dehydrogenase $(\mathrm{ODH})$ genes were used to elaborate the genetic structure collected from eight localities ranging from the northern to the southern coast of China. In total, 118 individuals for the RD gene and 108 for the $\mathrm{ODH}$ were sequenced. Overall (RD and $\mathrm{ODH}$ ) genes resulted in high $(0.741 \pm 0.032 ; 0.805 \pm 0.038)$ haplotype and low nucleotide $(0.01261 \pm 0.00165 ; 0.00747 \pm 0.00086)$ diversity. Molecular variance displayed higher values among the populations and lower values within the population where the fixation index $\mathrm{F}_{\mathrm{ST}}$ denoted 0.880 and 0.584 in $\mathrm{RD}$ and $\mathrm{ODH}$ genes respectively. The Dongshan population clustered separately in a phylogenetic tree as in the haplotype networking assessment. The current data suggests that the Dongshan population needs separate management.
\end{abstract}

\section{Keywords}

Cephalopoda, China Coast, Nuclear DNA, Octopuses

Copyright Faiz Muhammad et al. This is an open access article distributed under the terms of the Creative Commons Attribution License (CC BY 4.0), which permits unrestricted use, distribution, and reproduction in any medium, provided the original author and source are credited. 


\section{Introduction}

The class Cephalopoda embraces animals which are exclusively marine inhabitants. They have immense commercial and ecological significance, including a profound contribution as a source of protein for humans. The cephalopod has lobed and folded brain like that of vertebrates and are ingenious, migrant and largest of all molluscs (Mather and Kuba 2013; Cheng et al. 2013; Larson et al. 2015) and it is sensitive to environmental factors (Emery et al. 2016; Wang and Zheng 2017

Among cephalopods, the octopuses contribute $33 \%$ to the existing cephalopod assembly. Three hundred species of octopus are thought to exist along the coastal waters of Korea, China, and Japan (Roper et al. 1984; Norman and Sweeney 1997; Kang et al. 2012). One hundred thirty-four species are reported only in Chinese waters including the profit-making species like Octopus minor, Amphioctopus fangsiao, and Cistopus chinensis (Lu et al. 2012).

Previously, mtDNA molecular markers were popular because of their high mutation rate, maternal inheritance, and non-recombination (Vaseeharan et al. 2013). In the last decade, the nuclear DNA markers have been widely used for various investigations including identification, population genetics, comparisons between wild and captive populations, demographic evaluations, and rehabilitation projects (Chauhan and Rajiv 2010).

Carlini et al. (2000) was first who used nuclear DNA (Actin) for phylogenetic analysis of coleoid species subsequently other authors continued nuclear approach of investigation such as, (Warnke et al. 2003; Lindgren et al. 2004; Strugnell et al. 2005). The nuclear gene provides more information than mtDNA genes (Graybeal 1994). The rhodopsin and octopine dehydrogenase genes were used for phylogenetic and phylogeographic analysis of octopuses (Strugnell and Nishiguchi 2007; Toussaint et al. 2012). However, meagre information is available on population genetics of octopuses using $\mathrm{RD}$ and $\mathrm{ODH}$ genes. The $\mathrm{RD}$ and $\mathrm{ODH}$ genes are less complex and can provide better results than $18 \mathrm{~S}$ rDNA. Our present study aims to focus on collecting fundamental information about the population structure of this species using nuclear genes.

\section{Materials and methods}

Samples were collected from eight locations (Fig. 1). Thereafter, they were preserved in 95\% ethanol and transported to the laboratory. The total genomic DNA was isolated from muscle tissues using a standard protocol. Target genes were amplified by PCR using primers (Table 1). The total $25 \mu \mathrm{l}$ PCR mixture included (DNA template $1.25 \mu \mathrm{l}$, each primer $1.25 \mu \mathrm{l}$, ES Taq $12.5 \mu \mathrm{l}$, and $8.75 \mu \mathrm{l}$ water). Thermocycler conditions were as follows: denaturation at $94^{\circ} \mathrm{C}$ for $5 \mathrm{~min}, 35$ cycles $94^{\circ} \mathrm{C}$ for $30 \mathrm{~s}$, annealing at $55^{\circ} \mathrm{C}$ for $30 \mathrm{~s}$, extension at $72{ }^{\circ} \mathrm{C}$ for 30 s, and the final extension at $72{ }^{\circ} \mathrm{C}$ for $7 \mathrm{~min}$. Electrophoresis was performed on a $1.2 \%$ agarose gel and was sequenced using the same oligonucleotide primers. Sequences were aligned using MEGA 6 software (Tamura et al. 2013). Analysis of genetic 


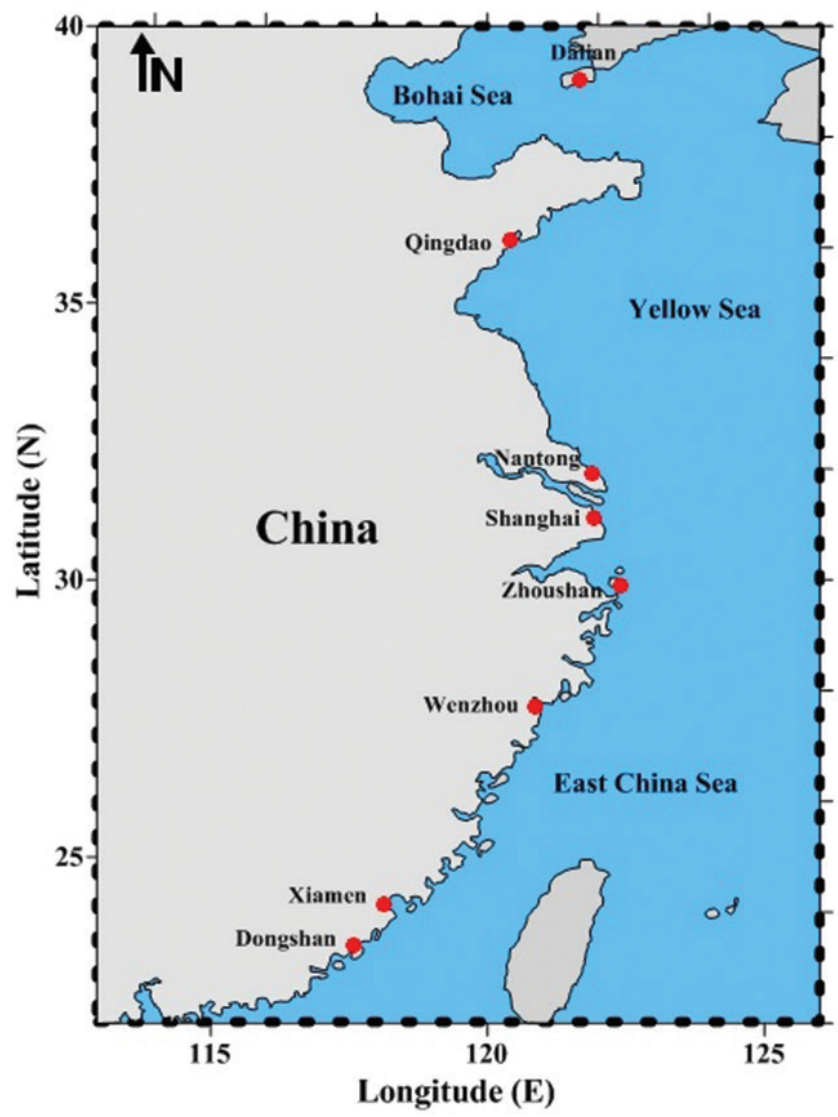

Figure I. Map showing the collection locations.

Table I. Oligonucleotide primers used for the amplification of nuclear rhodopsin and octopine dehydrogenase genes in Octopus minor.

\begin{tabular}{l|c|c|c|c}
\hline \multicolumn{1}{c|}{ Gene } & Primer and sequence (5'-3') & Tm $(\mathbf{C})$ & size (bp) & Reference \\
\hline \multirow{2}{*}{ Rhodopsin } & RDF 5'-GCTTTCCTCATGATTATC-3' & 50 & 653 & Toussaint et al. 2012 \\
\cline { 2 - 5 } & RDR 5'-TTCTCCATCATTGCCATC-3' & & & \\
\hline $\begin{array}{l}\text { Octopine } \\
\text { dehydrogenase }\end{array}$ & ODHF 5'-AAATCCCGACCAAACATG-3' & 50 & 618 & Toussaint et al. 2012 \\
\cline { 2 - 6 } & ODHR 5'-GTTAAGTTTGTACCAGTC-3' & & & \\
\hline
\end{tabular}

differentiation, AMOVA, molecular diversity indices, genetic differentiation values, and $\mathrm{F}_{\mathrm{ST}}$ values were determined with the ARLEQUIN software (Excoffier and Lischer 2010). Calculations of gene flow $\left(\mathrm{N}_{\mathrm{m}}\right)$ were performed using formula $N_{m}=\left(1-\mathrm{F}_{\mathrm{ST}}\right) / 2 \mathrm{~F}_{\mathrm{ST}}$. Haplotype and nucleotide diversity were estimated using DnaSP (Librado and Rozas 2009). The neighbor joining tree was constructed to check the genetic relationship between populations using MEGA 6 (Tamura et al. 2013). The haplotype networking was created using NETWORK software version 5.0.0.1 (Bandelt et al. 1999). 


\section{Results}

Rhodopsin Gene (RD): A fragment of $637 \mathrm{bp}$ of $\mathrm{RD}$ was sequenced from 118 individuals. RD gene showed 33 polymorphic sites and 13 haplotypes. Haplotype diversity (HD) ranged from 0.090 to 0.833 , nucleotide diversity $(\pi)$ remained in the lower range $(0.00027-0.001)$, where the average number of differences $(\mathrm{k})$ ranged from 0.166 to 1.388 (Table 2). Molecular variance revealed a higher percentage among the populations $(88.04 \%)$ while lower values were retrieved within $(11.96 \%)$ populations; fixation index $\mathrm{F}_{S T}$ was 0.880 (Table 3). The pairwise $\mathrm{F}_{S T}$ values ranged from 0.014 to 0.989. The lowest gene flow was observed in Dongshan population (Table 4). The values of Tajima's D tests generally showed negative values with exception to Xiamen population. The Wenzhou and Dongshan populations were statistically significant

Table 2. Genetic diversity parameters for $\mathrm{RD}$ and $\mathrm{ODH}$ sequences in Octopus minor.

\begin{tabular}{l|c|c|c|c|c|c}
\hline \multirow{4}{*}{ Gene } & Population & $\begin{array}{c}\text { Number of } \\
\text { segregating } \\
\text { sites }\end{array}$ & $\begin{array}{c}\text { Number of } \\
\text { ha2lotypes }\end{array}$ & $\begin{array}{c}\text { Haplotype } \\
\text { diversity } \\
(\mathbf{H d})\end{array}$ & $\begin{array}{c}\text { Nucleotide } \\
\text { diversity }(\boldsymbol{\pi})\end{array}$ & $\begin{array}{c}\text { Average } \\
\text { number of } \\
\text { differences (k) }\end{array}$ \\
\hline \multirow{5}{*}{$\begin{array}{l}\text { Rhodopsin } \\
\text { (RD) }\end{array}$} & Dalian & 3 & 4 & 0.676 & 0.001 & 0.819 \\
\cline { 2 - 7 } & Dongshan & 4 & 2 & 0.090 & 0.001 & 0.363 \\
\cline { 2 - 7 } & Nantang & 6 & 6 & 0.760 & 0.001 & 1.150 \\
\cline { 2 - 7 } & Qingdao & 2 & 2 & 0.404 & 0.001 & 0.426 \\
\cline { 2 - 7 } & Shanghai & 1 & 2 & 0.250 & 0.00040 & 0.250 \\
\cline { 2 - 7 } & Wenzhou & 1 & 2 & 0.166 & 0.00027 & 0.166 \\
\cline { 2 - 7 } & Xiamen & 4 & 5 & 0.833 & 0.002 & 1.388 \\
\cline { 2 - 7 } & Zhoushan & 2 & 3 & 0.292 & 0.0004 & 0.304 \\
\hline \multirow{5}{*}{$\begin{array}{l}\text { Octopine de- } \\
\text { hydrogenease } \\
(\text { ODH) }\end{array}$} & Dalian & 7 & 7 & 0.758 & 0.003 & 2.164 \\
\cline { 2 - 7 } & Dongshan & 4 & 5 & 0.775 & 0.002 & 1.341 \\
\cline { 2 - 7 } & Nantang & 8 & 8 & 0.828 & 0.003 & 2.076 \\
\cline { 2 - 7 } & Qingdao & 6 & 6 & 0.647 & 0.003 & 1.800 \\
\cline { 2 - 7 } & Whanghai & 6 & 6 & 1.000 & 0.004 & 2.733 \\
\cline { 2 - 7 } & Xenzhou & 2 & 2 & 0.222 & 0.001 & 0.444 \\
\cline { 2 - 7 } & Zhoushan & 5 & 6 & 0.717 & 0.001 & 0.897 \\
\hline
\end{tabular}

Table 3. Analysis of molecular variance of Octopus minor using RDand ODH.

\begin{tabular}{|c|c|c|c|c|c|}
\hline Gene & Source of variation & df & Sum of squares & variance component & Percentage $(\%)$ \\
\hline \multirow{4}{*}{$\mathrm{RD}$} & Among population & 8 & 573.404 & $5.57703 \mathrm{Va}$ & 88.04 \\
\hline & Within population & 110 & 83.334 & $0.75758 \mathrm{Vb}$ & 11.96 \\
\hline & Total & 118 & 656.737 & 6.33461 & \\
\hline & Fixation Index FST: & \multicolumn{4}{|c|}{0.880} \\
\hline \multirow{4}{*}{$\mathrm{ODH}$} & Among population & 8 & 174.799 & $1.79694 \mathrm{Va}$ & 58.44 \\
\hline & Within population & 99 & 126.490 & $1.27768 \mathrm{Vb}$ & 41.56 \\
\hline & Total & 107 & 301.290 & 3.07462 & \\
\hline & Fixation Index FST: & \multicolumn{4}{|c|}{0.584} \\
\hline
\end{tabular}


$(\mathrm{P}<0.05)$. Fu's Fs values were positive and statistically non-significant in all populations (Table 6). The phylogenetic analysis of eight populations separated Dongshan population with $99 \%$ bootstrap support (Fig. 2). The networking analysis revealed that haplotype three (Hap 3) was shared by six populations (Dalian, Nantong, Zhoushan, Qingdao, Shanghai, and Xiamen), Hap 4 was contributed by five populations (Dalain, Nantong, Qingdao, Xiamen, Zhoushan), Hap 2 shared between Dalian, Zhoushan, and Nantong, while Hap 7 appeared in two populations (Qingdao, Nantong), Hap 11 and Hap 12 were present in the Wenzhou and Xiamen populations, and Hap 5 and Hap 6 were independently represented in the Dongshan population (Fig. 4). Octopine dehydrogenase $(O D H)$ : A fragment of 597 bp was sequenced from 108 individuals, revealed 27 polymorphic sites and 29 haplotypes. Haplotype diversity remained higher $(0.222-1.000)$ than nucleotide diversity $(\pi)(0.001-0.004)$. The average number of differences $(\mathrm{k})$ ranged from 0.444 to 2.733 (Table 2). Molecular variance was revealed to be higher among the populations (58.44\%) while lower within a population $(41.56 \%)$. Fixation index $\left(\mathrm{F}_{\mathrm{ST}}\right)$ was determined as 0.584 (Table 3$)$. The pairwise $\mathrm{F}_{\mathrm{ST}}$ values ranged from 0.018 to 0.925 . The lowest gene flow was observed in Dongshan population whereas highest $\mathrm{N}_{\mathrm{m}}$ values were shown between Wenzhou and Xiamen populations (Table 5). Tajima's D showed negative values except in Dongshan, Shanghai, and Zhoushan populations whereas only Xiamen population was statistically significant $(\mathrm{P}<0.05)$. Fu's Fs presented negative values except Dongshan, Qingdao, and Wenzhou populations while only Shanghai and Xiamen populations were statistically

Table 4. Pairwise $\mathrm{F}_{\mathrm{ST}}$ below diagonal and gene flow $\left(\mathrm{N}_{\mathrm{m}}\right)$ values above diagonal RD gene.

\begin{tabular}{l|c|c|c|c|c|c|c|c}
\hline & Dalian & Dongshan & Nantong & Qingdao & Shanghai & Wenzhou & Xiamen & Zhoushan \\
\hline Dalian & - & 0.012 & 15.125 & 35.214 & 4.654 & 0.071 & 7.436 & $\infty$ \\
\hline Dongshan & 0.975 & - & 0.016 & 0.008 & 0.006 & 0.005 & 0.018 & 0.007 \\
\hline Nantong & 0.032 & 0.968 & - & 1.112 & 0.2 & 0.298 & $\infty$ & 2.996 \\
\hline Qingdao & 0.014 & 0.983 & 0.043 & - & 7.696 & 0.149 & 7.564 & 19.5 \\
\hline Shanghai & 0.097 & 0.987 & 0.200 & 0.061 & - & 0.103 & 2.104 & 23.309 \\
\hline Wenzhou & 0.875 & 0.989 & 0.626 & 0.770 & 0.828 & - & 0.575 & 0.118 \\
\hline Xiamen & 0.063 & 0.964 & $\infty$ & 0.062 & 0.192 & 0.465 & - & 0.002 \\
\hline Zhoushan & $\infty$ & 0.986 & 0.143 & 0.025 & 0.021 & 0.809 & 0.154 & - \\
\hline
\end{tabular}

Table 5. Pairwise $\mathrm{F}_{S T}$ below diagonal and gene flow $\left(\mathrm{N}_{\mathrm{m}}\right)$ values above diagonal ODH gene.

\begin{tabular}{l|c|c|c|c|c|c|c|c}
\hline & Dalian & Dongshan & Nantong & Qingdao & Shanghai & Wenzhou & Xiamen & Zhoushan \\
\hline Dalian & - & 0.086 & 0.884 & 14.2 & $\infty$ & 2.530 & 3.632 & $\infty$ \\
\hline Dongshan & 0.853 & - & 0.086 & 0.075 & 0.107 & 0.040 & 0.050 & 0.082 \\
\hline Nantong & 0.018 & 0.852 & - & $\infty$ & $\infty$ & 1.836 & 2.441 & 17.357 \\
\hline Qingdao & 0.034 & 0.869 & $\infty$ & - & 9.303 & 3.885 & 4.261 & 23.309 \\
\hline Shanghai & $\infty$ & 0.823 & $\infty$ & 0.051 & - & 1.702 & 2.406 & $\infty$ \\
\hline Wenzhou & 0.165 & 0.925 & 0.214 & 0.114 & 0.227 & - & 25.815 & 6.852 \\
\hline Xiamen & 0.121 & 0.908 & 0.170 & 0.105 & 0.172 & 0.019 & - & 12.32 \\
\hline Zhoushan & $\infty$ & 0.859 & 0.028 & 0.021 & $\infty$ & 0.068 & 0.039 & - \\
\hline
\end{tabular}




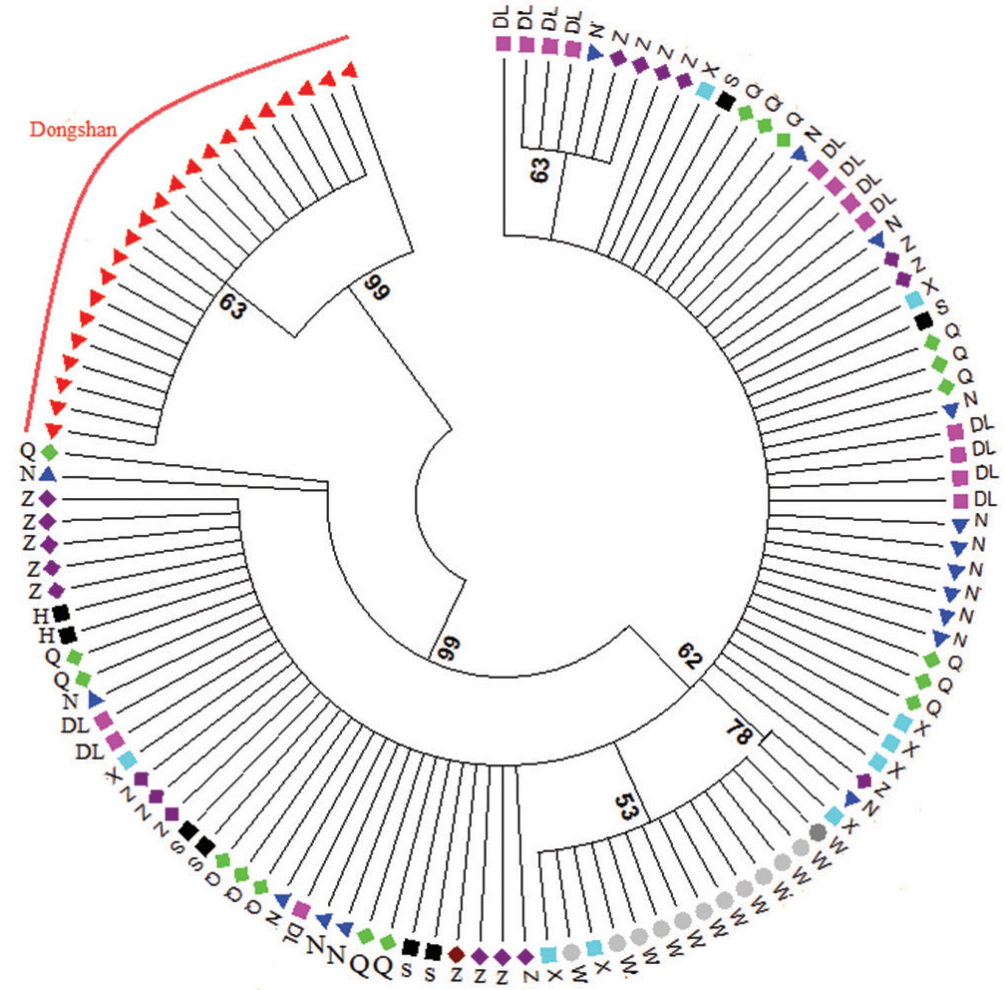

Figure 2. Neighbour-joining phylogenetic tree constructed based on RD gene sequences. Key: $D L=D a l i a n$, $\mathrm{N}=$ Nantong, $\mathrm{Q}=$ Qingdao, $\mathrm{S}=$ Shanghai, $\mathrm{W}=$ Wenzhou, $\mathrm{X}=$ Xiamen, $\mathrm{Z}=\mathrm{Zhoushan}$.

Table 6. Tajima's D and Fu's FS tests, corresponding $p$ value for eight population of $O$. minor.

\begin{tabular}{c|c|c|c|c|c}
\hline \multirow{3}{*}{ Gene } & \multirow{2}{*}{ Population } & \multicolumn{2}{|c|}{ Tajima's D } & \multicolumn{2}{c}{ Fu's Fs } \\
\cline { 2 - 6 } & & D & $\boldsymbol{p}$ & Fs & $\boldsymbol{p}$ \\
\hline \multirow{4}{*}{ RD } & Dalian & -0.260 & 0.449 & 1.447 & 0.805 \\
\cline { 2 - 6 } & Dongshan & -2.139 & 0.001 & 3.781 & 0.939 \\
\cline { 2 - 6 } & Nantang & -0.390 & 0.385 & 0.017 & 0.513 \\
\cline { 2 - 6 } & Qingdao & -0.416 & 0.405 & 1.360 & 0.789 \\
\cline { 2 - 6 } & Shanghai & -1.054 & 0.213 & 1.414 & 0.688 \\
\cline { 2 - 6 } & Wenzhou & -1.629 & 0.017 & 1.558 & 0.752 \\
\cline { 2 - 6 } & Xiamen & 0.061 & 0.533 & 0.087 & 0.518 \\
\cline { 2 - 6 } & Zhoushan & -1.125 & 0.167 & 0.934 & 0.666 \\
\hline \multirow{5}{*}{ ODH } & Dalian & -0.455 & 0.335 & -0.66426 & 0.345 \\
\cline { 2 - 6 } & Dongshan & 0.353 & 0.694 & 0.45027 & 0.612 \\
\cline { 2 - 6 } & Nantang & -0.220 & 0.445 & -0.77911 & 0.367 \\
\cline { 2 - 6 } & Qingdao & -0.031 & 0.526 & 0.00070 & 0.532 \\
\cline { 2 - 6 } & Shanghai & 0.665 & 0.741 & -2.73435 & 0.021 \\
\cline { 2 - 6 } & Wenzhou & -1.512 & 0.057 & 2.30182 & 0.843 \\
\cline { 2 - 6 } & Xiamen & -1.579 & 0.027 & -2.26025 & 0.022 \\
\cline { 2 - 6 } & Zhoushan & 0.579 & 0.300 & -0.95837 & 0.265 \\
\hline
\end{tabular}




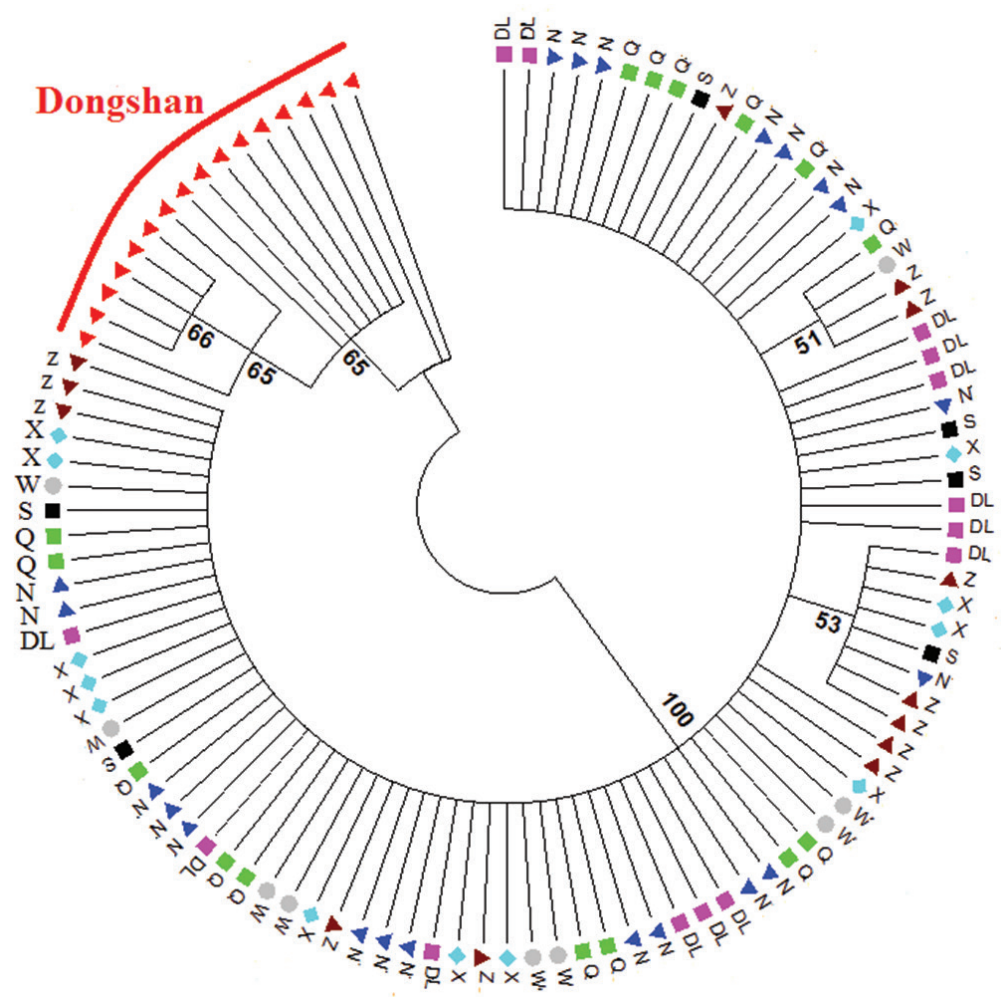

Figure 3. Neighbour-joining tree constructed based on the $\mathrm{ODH}$ gene. Key: $\mathrm{DL}=\mathrm{Dalian}, \mathrm{N}=\mathrm{Nantong}$, $\mathrm{Q}=$ Qingdao, $\mathrm{S}=$ Shanghai, $\mathrm{W}=$ Wenzhou, $\mathrm{X}=$ Xiamen, $\mathrm{Z}=$ Zhoushan.

significant (Table 6). The neighbour-joining phylogenetic tree described two distinct clades where Dongshan population clustered separately with 100\% bootstrap support (Fig. 3). The median joining network analysis described that Hap1 shared by seven populations followed by Hap2, which appeared in four populations, namely Dalian, Nantong, Qingdao, and Zhoushan. Hap 13 contributed by three populations (Dalian, Shanghai, and Zhoushan) similarly Hap 6 appeared in Dalian, Shanghai and Xiamen populations, Hap 17 shared by Nantong, Zhoushan, and Xiamen populations, Hap 16 shared by Nantong and Xiamen, Hap 19 appeared in Qingdao and Shanghai populations while Hap, 25 shared between Zhoushan and Wenzhou populations. Hap 8-12 were independently representing Dongshan population (Fig. 5).

\section{Discussion}

Octopus minor is a bottom-dwelling inhabitant and its migration is limited. The dispersal capacity has immense influence on population genetics. Tag- recapture investigations of $O$. vulgaris reported to be restricted within one $\mathrm{km}$ from the point of release (Melis 


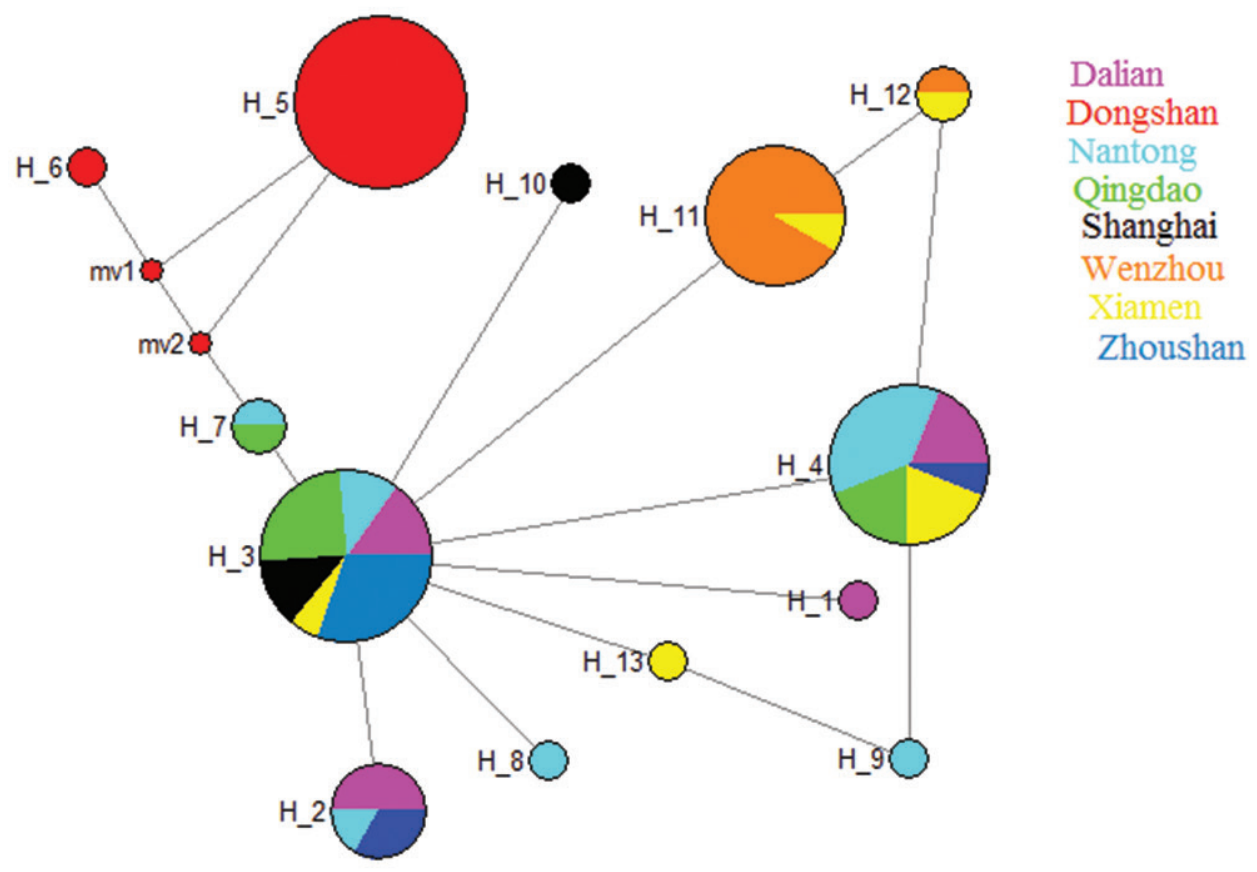

Figure 4. Median-joining networking drawn based on RD gene haplotypes. Colours represent the corresponding population frequencies. Key: Dalian; Dongshan; Nantong; Qingdao; Shanghai; Wenzhou; Xiamen; Zhoushan.

et al. 2018 and references therein). Conversely, dispersal range of $O$. minor juveniles and adults is not precisely known. The basic information of genetic variation and population structure is valuable for stocking, fisheries management, and conservation (Feng et al. 2017). Several divergent forces cause genetic differentiation, including geographic isolation, current and life history characteristics (Gao et al. 2016). In fact, oceanic processes are complex and a single reason cannot be claimed as source of divergent force. There are many islands and gulfs in China's sea, which can contribute to the gene flow complications of the populations (Gao et al. 2016). Earlier several studies were under taken including complete mitochondrial genome of this species (Cheng et al. 2012). Previous investigations on population genetics of $O$. minor present subtle to significant differences (Gao et al. 2016; Yang et al. 2015; Lü et al. 2013; Kang et al. 2012; Xu et al. 2011; Sun et al. 2010; Li et al. 2010). Our present analysis of two nuclear DNA gene sequences in $O$. minor collected from eight locations imparted variation. The haplotype diversity of $\mathrm{RD}$ gene ranged (0.090-0.833), the higher haplotype diversity was noted in Xiamen population and lowest haplotype diversity showed in Dongshan population. The ODH gene haplotype diversity was higher in Shanghai population (1.000) and was lowest in Wenzhou population (0.222). Comparative studies of Kang et al. (2012) between Korean and Chinese populations showed less haplotype diversity in Korean population than Chinese populations. However, only three Chinese populations were 


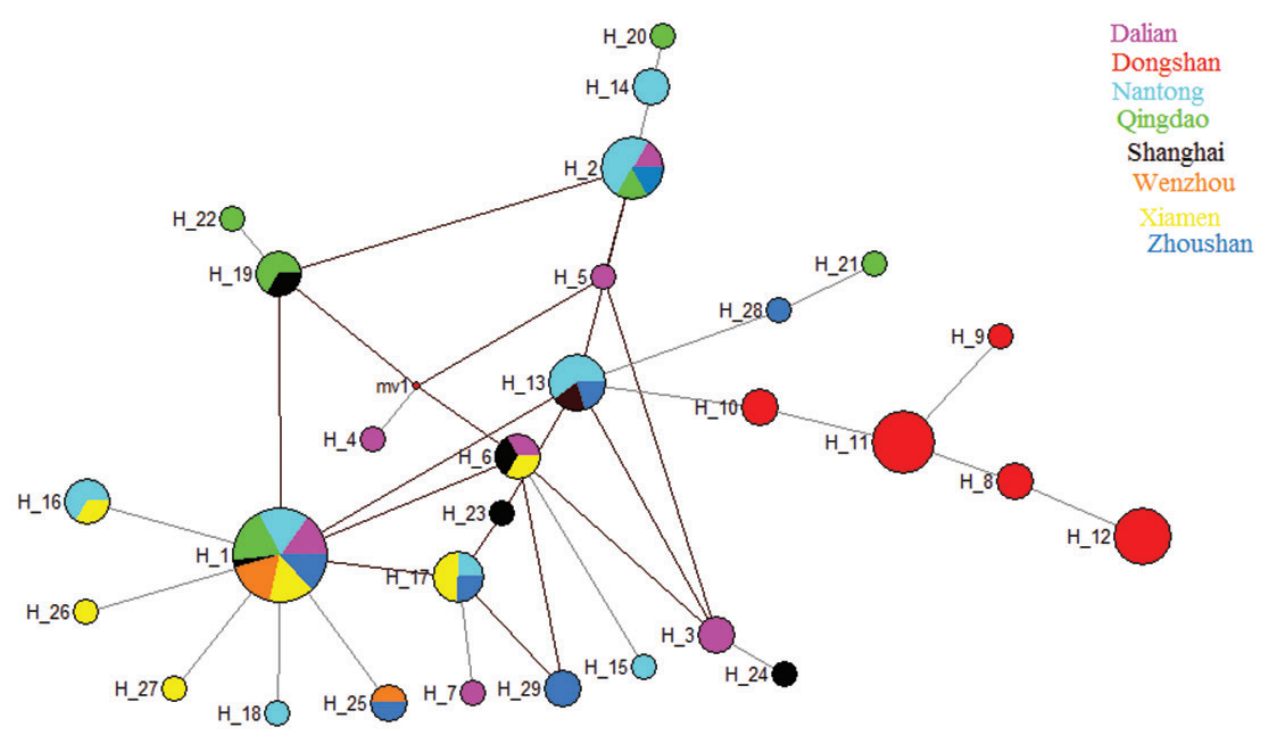

Figure 5. Median-joining networking drawn based on $\mathrm{ODH}$ gene haplotypes. Colours represent the corresponding population frequencies. Key: Dalian; Dongshan; Nantong; Qingdao; Shanghai; Wenzhou; Xiamen; Zhoushan.

sampled (Dalian, Tianjin, and Rongcheng) from Bohai and Yellow seas, which showed 0.43 to 0.64 values of haplotype diversity. This result is in line with present study. Chang et al. (2010) reported higher haplotype diversity in Lianyungang (0.934) and lowest (0.342) in Xiamen population but RD and ODH gene showed higher values $(0.833 ; 0.717)$ in Xiamen population. Yang et al. (2015) included five populations and reported that Qingdao has high diversity and more diverse than others but present research described high diversity of all northern populations (Table 2). The AMOVA results denoted higher values among populations and lower values within populations, where the fixation index $\mathrm{F}_{S \mathrm{~S}}$ was much higher. Yang et al. (2015) reported lower AMOVA values (10.88\%) between populations and higher values $(89.12 \%)$ within population, which is discordant. Higher $\mathrm{F}_{S T}$ values indicate a lower level of gene flow $\left(\mathrm{N}_{\mathrm{m}}\right)$ and higher genetic differentiation among populations (Hedrick and Goodnight 2005; Ye et al. 2015). The lowest gene flow between Dongshan with other counterpart populations was observed. $\mathrm{F}_{\mathrm{ST}}$ value of 0.05 is considered to indicate negligible genetic differentiation, while a value greater than 0.25 demonstrate high genetic differentiation within the analyzed population (Weir and Cockerham 1996). Based on this standard, the results obtained in this study showed high differentiation between Dongshan and other counterpart populations (Tables 4, 5).

The Tajima's D analysis of the RD gene showed negative values except for the Xiamen population, unlike to the results of the CO1 studies (Chang et al. 2010). The ODH gene revealed negative values for Tajima's D except for three populations, Shanghai, Zhoushan, and Dongshan. Results are similar to mtDNA COI investigations 
(Chang et al. 2010) where the Zhoushan population had positive values; Shanghai and Nantong populations were not included in COI studies. The RD gene revealed 13 haplotypes; among them Hap 3 was common, shared by 6 populations. None of the haplotypes of the Dongshan population was shared by other counterpart populations with similar COII and Cytb investigations (Lü et al. 2013; Li et al. 2013). Nevertheless, it differs with COII and Cytb (Lü et al. 2013; Li et al, 2013) where Wenzhou, Zhoushan, and Wenzhou, Xiamen populations remained isolated populations respectively. The ODH gene described 29 haplotypes among which Hap1 remained common, which was shared by seven populations. It is consistent with Lü et al. (2013) and $\mathrm{Li}$ et al. (2013) with respect to Dongshan population whereas differ with reference to Wenzhou and Xiamen populations. Studies of Kang et al. (2012) described sharing of one haplotype between Chinese and Korean populations. The neighbour-joining phylogenetic tree of both the genes distinctly clustered Dongshan population separately as in Cyt b, and COII studies (Li et al. 2013; Lü et al. 2013). However, COII unveiled Wenzhou and Zhoushan populations as separate clade (Lü et al. 2013); similarly, $16 \mathrm{~S}$ rRNA showed the Xiamen population as a separate clade (Li et al. 2010). Chang et al. (2010) reported Wenzhou and Xiamen populations as separate clade. Kang et al. (2012) investigated three clades where only one Chinese population (Dalian) parted as sub-clade along with Korean populations. Yang et al. (2015) using AFLP and Sun et al. (2010) using CO1 reported two clades, which is inconsistent with present study. The discrepancy of data is consequences of various unknown oceanic process, genetic markers used and range of sampling locations (Lü et al. 2013). To understand the data variation of $O$. minor along the Chinese coast, it is imperative to study the larval and adult dispersal range along with seasonal oceanic process during ontogenesis. Present data does not support the isolation by distance (IBD) because the geographic coastal distance between Dongshan and Xiamen is only $157 \mathrm{KM}$ while the gene flow between them is meagre as shown by $\mathrm{RD}$ and $\mathrm{ODH}(0.018 ; 0.050)$, whereas the coastal distance between Dalian and Xiamen is approximately $3700 \mathrm{Km}$ but the gene flow is higher (7.436; 3.632). Lü et al. (2013) was also noted gene flow discordance but the concrete reason is unknown. Melis et al. (2018) emphasized the causes of meagre larval dispersal in $O$. vulgaris including high mortality, philopatric behaviour of larvae and potential cryptic berries. Nevertheless, the above-mentioned factors are difficult to be disentangled, furthermore, oceanic fronts (temperature, salinity, density, turbidity, nutrients, velocity), upwelling and current systems can also influence the larval dispersal (Melis et al. 2018). It has long been entrenched that genetic structure of populations affected by glacier activities where sea level encountered climatic fluctuations during the Pleistocene period and caused gene flow restrictions in marine organisms (Imbrie et al. 1992). However, relatively high haplotype diversity reported in freshwater fish inhabiting nonglaciated regions or temperate regions (Bernatchez and Wilson 1998; Tan et al. 2015). Most of the studies related to $O$. minor gave high haplotype diversity in this region, including the present study.

Our present investigation has significant implications for conservation and favourable management of $O$. minor along the Chinese coast and it also shed light on the 
need for separate management of the Dongshan population: once an evolutionary lineage is lost, there is no possibility to be recover it (Mortiz 2002). We also recommend infield ontogenetic studies and larval migration ranges observations along with physical oceanographic parameters in future studies to understand the population genetics data inconsistency in $O$. minor reported along Chinese coastal waters.

\section{Acknowledgements}

This research was supported by the National Natural Science Foundation of China (NSFC) (41576131) and Talented Yong Scientist Program (PAK-15-012). We pay thanks to reviewers whose constructive comments helped us to improve this manuscript.

\section{References}

Bandelt HJ, Forster P, Rohl A (1999) Median-joining networks for inferring intraspecific phylogenies. Molecular Biology and Evolution 16: 37-48. https://doi.org/10.1093/oxfordjournals.molbev.a026036

Bernatchez L, Wilson CC (1998) Comparative phylogeography of Neartic and Paleartic fishes. Molecular Ecology 7(4): 431-452. https://doi.org/10.1046/j.1365-294x.1998.00319.x

Carlini DB, Reefe KS, Graves JE (2000) Actin gene family evolution and the phylogeny of coleoid cephalopods (Mollusca: Cephalopoda). Molecular Biology and Evolution 17: 1353-1370. https://academic.oup.com/mbe/article/17/9/1353/994836

Chauhan T, Rajiv K (2010) Molecular markers and their applications in fisheries and aquaculture. Advances in Bioscience and Biotechnology 1: 281-291. https://doi.org/10.4236/ abb.2010.14037

Chang KM, Li H, Lü ZM, Chi CF (2010) Genetic variation in different populations of Octopus variabilis in China coastal waters based on the COI gene analysis. Oceanologia et Limnologia Sinica 41: 307-314.

Cheng R, Zheng X, Lin X (2012) Determination of the complete mitochondrial DNA sequence of Octopus minor. Molecular Biology Reports 39(4): 3461-3470. https://link. springer.com/article/10.1007\%2Fs11033-011-1118-2

Cheng R, Zheng X, Ma Y, Li Q (2013) The complete mitochondrial genomes of two Octopods Cistopus chinensis and Cistopus taiwanicus: Revealing the phylogenetic position of Genus Cistopus within the order Octopoda. PLoS One 8(12): e84216. https://doi.org/10.1371/ journal.pone.0084216

Emery TJ, Hartmann K, Gardner C (2016) Management issues and options for small scale holobenthic octopus fisheries. Ocean and Coastal Management 120: 180-188. https://doi. org/10.1016/j.ocecoaman.2015.12.004

Excoffier L, Lischer HEL (2010) Arlequin suite ver 3.5: A new series of programs to perform population genetics analyses under linux and Windows. Molecular Ecology resources 10: 564-567. https://doi.org/10.1111/j.1755-0998.2010.02847.x 
Feng Y, Liu W, Xin X, Yang J, Wang W, Wei X, Liu X, Sun G (2017) Construction of a normalized full-length cDNA Library of Cephalopod Amphioctopus fangsiao and development of microsatellite markers. Journal of Ocean University of China 16(5): 897-904. https://link. springer.com/article/10.1007/s11802-017-3291-y

Gao X, Zheng X, Bo Q, Li Q (2016) Population genetics of the common long-armed Octopus Octopus minor (Sasaki, 1920) (Cephalopoda: Octopoda) in Chinese waters based on microsatellite analysis. Biochemical Systematics and Ecology 66: 129-136. https://doi. org/10.1016/j.bse.2016.03.014

Graybeal A (1994) Evaluating the phylogenetic utility of genes: a search for genes informative about deep divergences among vertebrates. Systematic Biology 43: 174-193. https://doi. org/10.1093/sysbio/43.2.174

Hedrick PW, Goodnight C (2005) A standardized genetic differentiation measure. Evolution 59(8):1633-8. https://doi.org/10.1111/j.0014-3820.2005.tb01814.x

Imbrie J, Boyle EA, Clemens SC, Duffy A, Howard WR, Kukla G, Kutzbach J, Martinson DG, Mclntyre A, Mix AC, Molfino B, Morley JJ, Peterson LC, Pisias NG, Prell WL, Raymo ME, Shackleton NJ, Toggweiler JR (1992) On the structure and origin of major glaciations cycles. I. Linear responses to Milankovitch forcing. Paleoceanography 7: 701-738. https:// doi.org/10.1029/92PA02253

Kang JH, Park JY, Choi TJ (2012) Genetic differentiation of octopuses from different habitats near the Korean Peninsula and eastern China based on analysis of the mDNA cytochrome C oxidase 1 gene. Genetic and Molecular Research 11(4): 3988-3997. https:// doi.org/10.4238/2012.November.21.2

Larson S, Ramsay C, James AC (2015) Multiple paternity and preliminary population genetics of giant pacific octopuses, Enteroctopus dofleini in Oregon, Washington and the Southeast coast of Vancouver Island, BC. Diversity 7: 195-205. https://doi.org/10.3390/d7020195

Librado P, Rozas J (2009) DnaSP v5: A software for comprehensive analysis of DNA polymorphism data. Bioinformatics 25: 1451-1452. https://doi.org/10.1093/bioinformatics/btp187

Li H, Zhenming Lü, Chang KM (2010) Genetic variation in different populations of Octopus variabilis in China coastal waters based on the $16 \mathrm{~S} r$ RNA gene analysis. Journal of Zhejiang Ocean University 29(4): 325-330.

Li HM, Zhenming Lü, Liu LQ, Wu CW, Zhang JS (2013) Genetic structure in 4 Octopus variabilis populations from China Coastal waters based on mitochondrial Cyt b Gene sequence. Oceanologia et Limnologia Sinica 44(3): 626-631.

Lindgren AR, Giribet G, Nishiguchi MK (2004) A combined approach to the phylogeny of Cephalopoda (Mollusca). Cladistics 20: 454-486. https://onlinelibrary.wiley.com/doi/ abs/10.1111/j.1096-0031.2004.00032.x

Lu CC, Zhang XD, Lin XZ (2012) Diversity of Cephalopoda from the waters of the Chinese mainland and Taiwan. In: Lin M, Wang CG (Eds) Proceedings of the $1^{\text {st }}$ Mainland and Taiwan symposium of marine biodiversity studies. Ocean Press, Beijing, 76-87.

Lü ZM, Liu LQ, Li H, Wu CN, Zhang JS (2013) Deep phylogeographic break among Octopus variabilis populations in China: Evidence from mitochondrial and nuclear DNA analyses. Biochemial Systematics and Ecology 51: 224-231. https://doi.org/10.1016/j.bse.2013.09.003 
Mather JA, Kuba MJ (2013) The cephalopod specialties: Complex nervous system, learning and cognition. Canadian Journal of Zoology 91: 431-449. https://doi.org/10.1139/cjz2013-0009

Melis R, Vacca L, Cuccu D, Mereu M, Cau A, Follesa MC, Cannas R (2018) Genetic population structure and phylogeny of the common octopus Octopus vulgaris Cuvier, 1797 in the Western Mediterranean Sea through nuclear and mitochondrial markers. Hydrobiologia 807: 277-296. https://link.springer.com/article/10.1007/s10750-017-3399-5

Mortiz C (2002) Strategies to protect biological diversity and the evolutionary processes that sustain it. Systematic Biology 51(2): 238 254. https://doi.org/10.1080/10635150252899752

Norman MD, Sweeney MJ (1997) The shallow water octopuses (Cephalopoda: Octopodidae) of the Philippines. Invertebrate Taxonomy 11: 89-140. https://doi.org/10.1071/IT95026

Roper CFE, Sweeney MJ, Nauen CE (1984) Cephalopods of the world. Food and Agriculture Organization Rome 3: 277.

Strugnell JM, Norman M, Jackson J, Drummond AJ, Cooper A (2005) Molecular phylogeny of coleoid cephalopods (Mollusca: Cephalopoda) using a multigene apparoach; the effect of data partitioning on resolving phylogenies in a Bayesian framework. Molecular phylogenetics and Evolution 37(2): 426-441. https://doi.org/10.1016/j. ympev.2005.03.020

Strugnell J, Nishiguchi MK (2007) Molecular phylogeny of coleoid cephalopods (Mollusca: Cephalopoda) inferred from three mitochondrial and six nuclear Loci: A comparison of alignment, implied alignment and analysis methods. Journal of Molluscan Studies 73: 399-410. https://doi.org/10.1093/mollus/eym038

Sun BC, Yang JM, Sun GH, Liu XQ, Liu LJ, Wang WJ, Zheng XD (2010) Sequence and molecular phylogeny of mitochondrial COI gene fragment in five populations of Octopus variabilis in China. Oceanologia et Limnologia Sinica 41: 259-265.

Tamura K, Stecher G, Peterson D, Filipski A, Kumar S (2013) MEGA6: Molecular Evolutionary Genetics Analysis version 6.0. Molecular Biology and Evolution 30: 2725-2729. https://doi.org/10.1093/molbev/mst197

Tan MP, Jamsari AFJ, Muchlisin ZA, Siti Azizah MN (2015) Mitochondrial genetic variation and population structure of the striped snakehead, Channa striata in Malaysia and Sumatra, Indonesia. Biochemical Systematics and Ecology 60: 99-105. https://doi.org/10.1016/j. bse.2015.04.006

Toussaint RK, Scheel D, Sage GK, Talbot SL (2012) Nuclear and mitochondrial markers reveal evidence for genetically segregated cryptic speciation in giant Pacific octopuses from Prince William Sound, Alaska. Conservation Genetics 13: 1483-1497. https://link.springer.com/ article/10.1007/s10592-012-0392-4

Vaseeharan B, Rajakamaran P, Jayaseelan D, Vincent AY (2013) Molecular markers and their application in genetic diversity of penaeid shrimp. Aquaculture International 21:219-241. https://doi.org/10.1007/s10499-012-9582-9

Wang JH, Zheng XD (2017) Comparison of the genetic relationship between nine Cephalopod species based on cluster analysis of karyotype evolutionary distance. Comparative Cytogenetics 11(3): 477-497. https://doi.org/10.3897/compcytogen.v11i3.12752 
Warnke K, Plotner J, Santana JI, Rueda MJ, LLina SO (2003) Reflections on the phylogenetic position of Spirula (Cephalopoda): preliminary evidence from the 18S ribosomal RNA gene. Berliner Palaobiologisch Abhandlungen 3: 253-260.

Weir BS, Cockerham CC (1996) Genetic data analysis II: Methods for discrete population genetic data. Sinauer Associates Inc., Sunderland, MA.

Xu MY, Li JJ, Guo BY, Lü ZM, Zhou C, Wu CW (2011) Genetic diversity of seven populations of Octopus variabilis in China's coastal waters based on the 12SrRNA and COIII gene analysis. Oceanologia et Limnologia Sinica 42: 387-396.

Ye YY, Wu CW, Li JJ (2015) Genetic population structure of Macridiscus multifarious (Mollusca: Bivalvia) on the basis of mitochondrial markers: strong population structure in a species with a short planktonic larval stage. PloS ONE 10(12): e 0146260. https://doi. org/10.1371/journal.pone.0146260 